\title{
MONITORIZAÇÃO DE MICROALGAS PLANCTÔNICAS POTENCIALMENTE TÓXICAS NA ÁREA DE MARICULTURA DA ENSEADA DE ARMAÇÃO DE ITAPOCOROY - PENHA - SC.
}

\author{
RÖRIG, L.R.; GUIMARÃES**, S.C.P.; LUGLI*, D.O.; PROENÇA, L.A.O.; MANZONI, G.C. \\ \& A.C. MARENZI
}

CTTMar - UNIVALI

Rua Uruguai, 458, Cx.P. 360, Itajaí, SC - CEP 88.302-202

RESUMO

\begin{abstract}
O presente trabalho trata-se de uma síntese de aproximadamente 17 meses de amostragens de fitoplâncton na área de maricultura da Enseada de Armação do Itapocoroy, município de Penha, Santa Catarina. Os objetivos foram conhecer as principais espécies de microalgas planctônicas ocorrentes no local, especialmente as espécies tóxicas ou potencialmente tóxicas; verificar as características das massas de água associadas às diferentes espécies ou assembléias de microalgas (salinidade, temperatura e transparência) e monitorar a ocorrência de densidades elevadas de espécies tóxicas ou potencialmente tóxicas para prevenir possíveis intoxicações pela ingestão de moluscos com acúmulo de ficotoxinas. Os resultados indicam a ocorrência de padrões sazonais de sucessão de espécies. Espécies potencialmente produtoras de toxinas diarréicas e amnésicas tendem a ocorrer nos meses de inverno e primavera. Entre o final da primavera e durante o verão a ocorrência de Trichodesmium spp. indica possibilidade de toxinas de cianobactérias. Alexandrium fraterculus - um potencial produtor de toxina paralisante - ocorreu em pequenas densidades e principalmente no inverno.
\end{abstract}

Palavras Chave: Microalgas Tóxicas, Monitoramento, Maricultura, Costa de Santa Catarina.

\section{MONITORING OF POTENTIALLY TOXIC MICROALGAE IN THE SHELLFISH CULTURE SITE OF ARMAÇÃO DO ITAPOCOROY BIGHT - PENHA - SC.}

\begin{abstract}
This paper is a descriptive synthesis of approximately 17 months of phytoplankton sampling in the shellfish culture area of Enseada de Armação do Itapocoroy (Penha - Santa Catarina Brazil). The goal of this program was to know the local phytoplankton composition, specially the toxic and potentially toxic ones; to verify the characteristics of water masses associated to the different phytoplankton assemblies and to monitor the occurrence of blooms of toxic or potentially toxic species, in order to prevent human intoxication by ingestion of contaminated shellfish. The results have indicated the occurrence of seasonal patterns in species succession. Potential producers of diarrhetic toxins tend to occur in winter and spring. From late spring to late summer the occurrence of Trichodesmium spp. indicate the possible presence of cyanobacteria toxins. Alexandrium fraterculus - a potential producer of paralytic toxin - has ocurred only in low cell densities and mainly in winter.
\end{abstract}

Key Words: Toxic Microalgae, Monitoring, Mussel Aquaculture, Santa Catarina Coast.

* bolsista ProBIC - FACIMAR, ProPPEx, UNIVALI

** bolsista PED (Projeto de Execução Descentralizada, Ministério do Meio Ambiente) - FACIMAR/ UNIVALI 


\section{INTRODUÇÃO}

A Enseada de Armação do Itapocoroy representa uma das principais áreas de cultivo de moluscos (mexilhões e ostras) de Santa Catarina (Figura 1). Segundo a Associação de Maricultores de Penha (AMAP), a produção anual na enseada atingiu 500 ton de mexilhões e 3000 dúzias de ostras em 1997. A maricultura no município de Penha iniciou-se e desenvolveu-se em função das atividades de pesquisa e extensão da Faculdade de $\mathrm{Ci}$ ências do Mar da Universidade do Vale do Itajaí (FACIMAR - UNIVALI) que conscientizaram a população local sobre as possibilidades econômicas da maricultura e transferiram tecnologia e assistência aos que interessaramse na atividade.

Com o acelerado crescimento da maricultura no local, surgiu a preocupação, por parte da FACIMAR - UNIVALI, em garantir a qualidade e produtividade das águas da enseada, uma vez que disso depende a qualidade do produto e a sustentabilidade da atividade. Diversos projetos foram então elaborados e desenvolvidos pela instituição, entre eles a determinação de variáveis físico-químicas da água (salinidade, temperatura, transparência, nutrientes, matéria orgânica e metais pesados); a monitorização dos níveis de coliformes fecais da água; a composição e biomassa de microalgas planctônicas (fitoplâncton) e a presença de ficotoxinas na carne dos moluscos cultivados. O desenvolvimento desses projetos fez com que a Enseada de Armação do Itapocoroy fosse a primeira área de maricultura no país a apresentar um sistema de monitorização e controle da qualidade das águas e do produto cultivado, assemelhandose a países como Chile, Espanha, França,

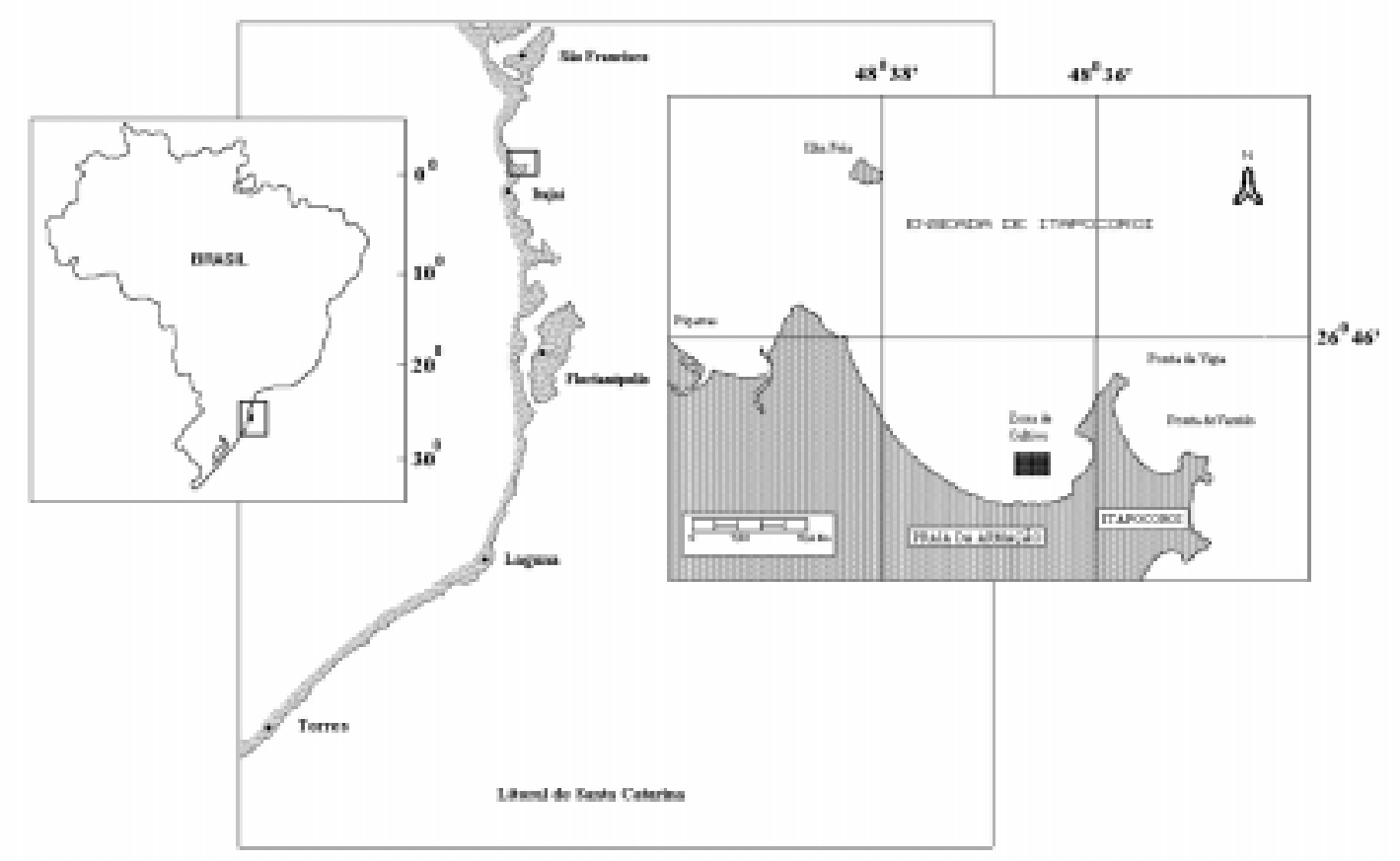

Figura 1. Localização da Enseada de Armação do Itapocoroy e da área de cultivo de moluscos onde foram coletadas as amostras. 
Japão e outros. Nesses países as atividades de maricultura historicamente apresentam elevada importância sócio-econômica, sendo amparadas por rigorosa legislação sanitária (Andersen, 1996), a qual é ainda incipiente no Brasil.

O presente trabalho trata especificamente de um importante problema relacionado ao desenvolvimento da maricultura: a possibilidade de acúmulo de toxinas na carne de bivalvos cultivados em função da ocorrência e ingestão, por parte dos bivalvos, de microalgas potencialmente produtoras dessas toxinas. O consumo desses moluscos por seres humanos pode gerar diversos efeitos tóxicos - de uma simples diarréia até a morte - os quais são conhecidos há muito tempo. Um dos primeiros casos fatais de envenenamento humano após o consumo de mexilhões contaminados com dinoflagelados tóxicos ocorreu em 1793, quando o Capitão George Vancouver e sua tripulação atracaram na Columbia Britânica, em uma área agora chamada de Poison Cove (= Enseada do Veneno) (Hallegraeff et al., 1995). Naquela região, os indígenas tinham como tabu não consumir fru- tos-do-mar quando a água estava fluorescente devido a florações de dinoflagelados (Dale \& Yentsch, 1978). Até 1995, cerca de 2000 casos de envenenamento humano por consumo de frutos-do-mar tinham sido reportados, sendo $15 \%$ fatais (Hallegraeff et al., 1995).

As ficotoxinas, como são chamadas as toxinas produzidas por algas, podem ser de diversos tipos em função do grupo de algas que as produz, da categoria química a que enquadram-se e dos sintomas por elas produzidos em seres humanos (Tabela 1).

Embora diversos casos históricos de intoxicação por consumo de moluscos tenham sido reportados por comunidades litorâneas de Santa Catarina (M. Polette, com. pess.), só existe um caso oficial documentado. Este registro trata-se da intoxicação de diversas pessoas que consumiram mexilhões de bancos naturais na localidade de Santo Antônio de Lisboa, em Florianópolis, no ano de 1991 (Zenebon \& Pregnolatto, 1992). A sintomatologia das vítimas, que incluiu fortes diarréias e dores abdominais, foi atribuída ao veneno diarréico de moluscos (VDM ou DSP diarrhetic shelfish poisoning), produzido por

Tabela 1. Diferentes grupo de ficotoxinas, seus organismos produtores, categoria química das toxinas e sintomas principais $(\mathrm{VDM}=$ veneno diarréico de moluscos; $\mathrm{VPM}=$ veneno paralisante de moluscos; $\mathrm{VAM}=$ veneno amnésico de moluscos; $\mathrm{VCM}=$ veneno ciguatérico de moluscos; $\mathrm{VNM}=$ veneno neurológico de moluscos; $\mathrm{VC}=\mathrm{veneno}$ de cianobactérias). Fonte: Hallegraeff et al. (1995).

\begin{tabular}{|c|c|c|c|}
\hline \hline $\begin{array}{c}\text { Tipo de } \\
\text { toxina }\end{array}$ & Organismos produtores & Categoria química & Sintomas principais \\
\hline VDM & $\begin{array}{c}\text { dinoflagelados: Dinophysis spp.; } \\
\text { Prorocentrum lima }\end{array}$ & Poliéteres & diarréia, náusea, vômito \\
\hline VPM & $\begin{array}{c}\text { dinoflagelados: Alexandrium spp.; } \\
\text { Gymnodinium catenatum; Pyrodinium } \\
\text { bahamense. }\end{array}$ & Alcalóides & $\begin{array}{c}\text { diarréia, náusea, vômito, } \\
\text { parestesia, parada respiratória }\end{array}$ \\
\hline VAM & diatomáceas: Pseudo-nitzschia spp & Amino-ácidos excitatórios & $\begin{array}{c}\text { diarréia, náusea, vômito, } \\
\text { parestesia, parada respiratória, } \\
\text { amnésia }\end{array}$ \\
\hline VCM & $\begin{array}{c}\text { dinoflagelados: Prorocentrum spp.; } \\
\text { Gambierdiscus toxicus; Ostreopsis spp. }\end{array}$ & Poliéteres & $\begin{array}{c}\text { diarréia, náusea, vômito, } \\
\text { parestesia, reversão de } \\
\text { temperatura }\end{array}$ \\
\hline VNM & dinoflagelados: Gymnodinium breve & Poliéteres & $\begin{array}{c}\text { diarréia, náusea, vômito, } \\
\text { parestesia, reversão de } \\
\text { temperatura, bronquiconstrição }\end{array}$ \\
\hline VC & $\begin{array}{c}\text { lesões e perturbações } \\
\text { neurológicas e hepáticas, } \\
\text { dermatite }\end{array}$ \\
\hline \hline
\end{tabular}


dinoflagelados do gênero Dinophysis, encontrados em densidades relativamente altas nas águas do local.

A possibilidade de presença de espécies potencialmente tóxicas nas águas costeiras de Santa Catarina, aliada ao aumento na produção e consumo de moluscos bivalvos no estado, levou-nos a crer em um provável aumento de intoxicações. Assim, dentro de uma intenção maior de preservar a sustentabilidade da maricultura no município de Penha e, por conseqüência, em todo o estado, realizamos este trabalho de monitorização, cujos objetivos específicos foram: conhecer as principais espécies de microalgas planctônicas ocorrentes ao longo do ano na área de maricultura da Enseada de Armação do Itapocoroy, especialmente no que refere-se às espécies tóxicas ou potencialmente tóxicas; verificar as características das massas de água associadas às diferentes espécies ou assembléias de microalgas (salinidade, temperatura e transparência) e monitorar a ocorrência de densidades elevadas de espécies tóxicas ou potencialmente tóxicas para prevenir possíveis intoxicações pela ingestão de moluscos com acúmulo de ficotoxinas.

\section{METODOLOGIA}

Amostragens de fitoplâncton foram realizadas em intervalos aproximadamente semanais entre fevereiro e dezembro de $1996 \mathrm{e}$ aproximadamente quinzenais entre janeiro e setembro de 1997, em um ponto localizado no interior da área de cultivo da Enseada de Armação do Itapocoroy (junto à corda da FACIMAR - UNIVALI) (Figura 1), utilizando-se um amostrador de coluna de água integrada (método tubing, ou mangueira, conforme Sournia, 1978). Este amostrador consistiu de uma mangueira plástica de uma polegada de diâmetro interno, com $5 \mathrm{~m}$ de comprimento (aproximadamente igual a profundidade do ponto de coleta) e dotada de válvula de fechamento superior. A mangueira era introduzida com a válvula aberta e, ao chegar próxima ao fundo, a válvula era fechada para reter a água dentro da mangueira. Desta maneira era obtida uma amostra representativa da coluna de água inteira. $O$ conteúdo da mangueira era então despejado em um balde e homogeneizado para depois retirar-se uma alíquota de $200 \mathrm{ml}$, a qual era fixada em solução de lugol a $2 \%$. As amostras foram analisadas em microscópio invertido segundo o método de Utermöhl (1958), sendo contados ao nível de espécie ou gênero, somente os organismos considerados potencialmente tóxicos. Os demais organismos foram agrupados ao nível de classe (Dinoflagelados Dinophyceae e Diatomáceas Bacillariophyceae) ou outros grupos. Paralelamente, foram tomadas amostras qualitativas com rede de plâncton de malha $20 \mu \mathrm{m}$, as quais foram utilizadas em procedimentos de identificação taxonômica e ainda dados de salinidade (refratômetro), temperatura da água (termômetro de mercúrio) e transparência (profundidade do disco de Secchi). A identificação das espécies foi realizada com base em Balech (1988), Cardoso (1993), Desikachary (1959), Steindinger \& Williams (1970) e Tomas (1997).

\section{RESULTADOS E DISCUSSÃO}

Em relação às características físicoquímicas das águas da enseada, constatouse que a salinidade raramente é menor do que 30 , e as temperaturas variam entre 16 e $28^{\circ} \mathrm{C}$, com um claro padrão sazonal (Figura 2). A baixa profundidade média do local determina ausência de estratificação significativa na coluna de água ao longo do ano. Os valores de transparência da água foram predominantemente maiores do que $2,5 \mathrm{~m}$ ( $75 \%$ dos dias amostrados) (Figura 2), sendo que com este valor ou maior, existe luz em toda a coluna de água, fato que implica, por exemplo, na possibilidade de desenvolvimento de algas bentônicas. 
O fitoplâncton da Enseada da Armação do Itapocoroy mostrou-se dominado por diatomáceas ao longo de todo o período amostrado, sendo que a importância relativa de dinoflagelados aumentou nos meses de inverno e primavera, o que pode ser constatado pelas menores razões Diatomáceas: Dinoflagelados nesta época (Figura 3 e células hachuradas na Tabela 2). Entre os outros grupos ocorrentes, destacam-se as cianobactérias do gênero Trichodesmium, que florescem na costa catarinense entre os meses de novembro e abril, podendo também ser tóxicas (Guimarães e Rörig, 1997; Rörig et al., 1998).

Em termos de densidades de células em geral, os maiores valores foram observados na primavera, coincidindo com as típicas florações que ocorrem nesta época em latitudes médias e altas. Entre as diatomáceas, as espécies potencialmente tóxicas do gênero Pseudo-nitzschia ficaram entre as mais abundantes (Tabela 3). Em relação aos dinoflagelados potencialmente tóxicos, destacam-se como mais comuns as espécies dos gêneros Dinophysis e Prorocentrum.

Estes resultados preliminares indicam que esta área pode sofrer problemas com toxinas do tipo VDM - produzidas por Dinophysis spp. e Prorocentrum spp. e também do tipo

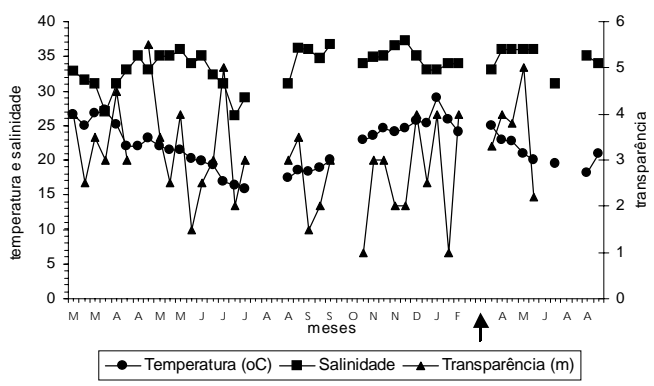

Figura 2. Valores de temperatura $\left({ }^{\circ} \mathrm{C}\right)$ da água, salinidade e transparência da água $(\mathrm{m})$ nas amostragens entre fevereiro de 1996 e setembro de 1997. A seta no eixo " $X$ " indica um período de 2 meses de ausência de amostragens (entre fevereiro e maio de 1997).
VAM em função da ocorrência de Pseudonitzschia spp. O aparecimento de Trichodesmium spp. indica ainda a possibilidade de problemas com toxinas de cianobactérias, que podem ser neurotóxicas. Em termos de épocas propícias, pode-se sugerir que problemas com VDM podem ser esperados especialmente no inverno e primavera, épocas onde as densidades de Prorocentrum spp. e Dinophysis spp. são mais significativas. Problemas com VAM estariam mais restritos aos meses de primavera devido às altas densidades de Pseudo-nitzschia spp. nessa época e problemas com toxinas de cianobactérias podem ser esperados entre o final da primavera e final do verão, época de aparecimento de florações de Trichodesmium spp. Em algumas ocasiões (especialmente no inverno) já foi constatado na enseada e em áreas adjacentes, densidades baixas de Alexandrium fraterculus, um potencial produtor de toxinas do tipo VPM. Em relação às toxinas VAM é necessário um estudo mais aprofundado da taxonomia das espécies de Pseudo-nitzschia para supor a sua ocorrência. Amostragens anteriores e de outras áreas do Estado de Santa Catarina já confirmaram a ocorrência de $P$. pungens e $P$. pseudodelicatissima, a primeira sendo potencialmente tóxica e segunda não tóxica (Rörig,

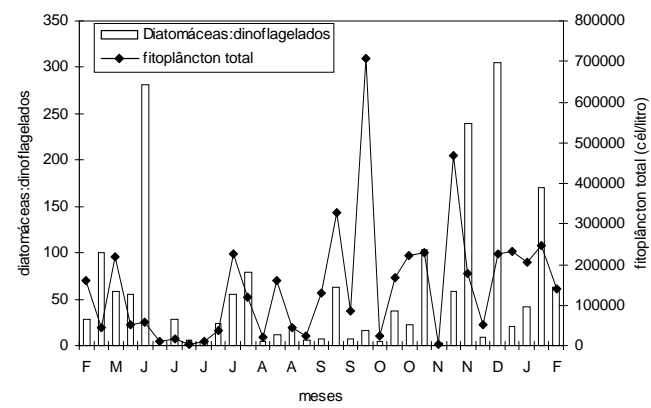

Figura 3. Razão diatomáceas:dinoflagelados e densidade de células de fitoplâncton total em células por litro (cél./litro) entre fevereiro de 1996 e fevereriro de 1997. 
RÖRIG et al.: Monitorização de microalgas tóxicas.

Tabela 2. Densidade (cel./l) de dinoflagelados, diatomáceas e outros grupos de microalgas planctônicas coletadas nas amostras pelo método tubing e razão Diatomáceas : Dinoflagelados (as células hachuradas destacam razões menores do que 30 , valor adotado como limite entre razões altas e baixas para fins ilustrativos). Obs.: n/d= não determinado; $(-)=$ ausência do dado.

\begin{tabular}{|c|c|c|c|c|}
\hline DATA & "Dinoflagelados & "Diatomáceas & "Outros Grupos & "Diatom./Dinofl \\
\hline 16.02 .96 & 5408 & 155035 & 902 & 28,7 \\
\hline 18.05 .96 & 440 & 44200 & 960 & 100,5 \\
\hline 25.05 .96 & 3680 & 214176 & 0 & 58,2 \\
\hline 29.05 .96 & 880 & 48800 & 40 & 55,5 \\
\hline 05.06 .96 & 200 & 56360 & 80 & 281,8 \\
\hline 12.06 .96 & 11776 & $n / d$ & 0 & - \\
\hline 22.06 .96 & 600 & 17120 & 0 & 28,5 \\
\hline 04.07 .96 & 300 & 1720 & 60 & 5,7 \\
\hline 10.07 .96 & 1960 & 8440 & 0 & 4,3 \\
\hline 17.07 .96 & 1440 & 34960 & 0 & 24,3 \\
\hline 31.07 .96 & 4048 & 221168 & 368 & 54,6 \\
\hline 02.08 .96 & 1472 & 117760 & 0 & 80,0 \\
\hline 06.08 .96 & 3680 & 18320 & 0 & 5,0 \\
\hline 13.08 .96 & 12880 & 148304 & 0 & 11,5 \\
\hline 23.08 .96 & 2253 & 39659 & 1802 & 17,6 \\
\hline 24.08 .96 & 3160 & 19640 & 280 & 6,2 \\
\hline 07.09 .96 & 13984 & 114816 & 2576 & 8,2 \\
\hline 14.09 .96 & 5152 & 323472 & 368 & 62,8 \\
\hline 21.09 .96 & 9568 & 75072 & 1472 & 7,8 \\
\hline 28.09 .96 & 39430 & 665879 & 1877 & 16,9 \\
\hline 12.10 .96 & 4400 & 19280 & 0 & 4,4 \\
\hline 26.10 .96 & 4440 & 163024 & 0 & 36,7 \\
\hline 29.10 .96 & 9464 & 213623 & 451 & 22,6 \\
\hline 02.11 .96 & 2208 & 227056 & 368 & 102,8 \\
\hline 10.11 .96 & 860 & 2120 & 320 & 2,5 \\
\hline 23.11 .96 & 7885 & 459693 & 0 & 58,3 \\
\hline 30.11 .96 & 736 & 175904 & 368 & 239,0 \\
\hline 07.12 .96 & 5160 & 45000 & 640 & 8,7 \\
\hline 14.12 .96 & 736 & 224848 & 0 & 305,5 \\
\hline 18.12 .96 & 10336 & 223540 & 0 & 21,6 \\
\hline 15.01 .97 & 4784 & 200928 & 736 & 42,0 \\
\hline 24.01 .97 & 1427 & 244352 & 0 & 171,2 \\
\hline 15.02 .97 & 2208 & 138368 & 368 & 62,7 \\
\hline 16.04 .97 & 14433 & $\mathrm{n} / \mathrm{d}$ & $\mathrm{n} / \mathrm{d}$ & - \\
\hline 30.04 .97 & 6320 & $\mathrm{n} / \mathrm{d}$ & $\mathrm{n} / \mathrm{d}$ & - \\
\hline 15.05 .97 & 1000 & $n / d$ & $\mathrm{n} / \mathrm{d}$ & - \\
\hline 27.05 .97 & 2020 & $n / d$ & $n / d$ & - \\
\hline 10.06 .97 & 580 & $\mathrm{n} / \mathrm{d}$ & $\mathrm{n} / \mathrm{d}$ & - \\
\hline 24.06 .97 & 5740 & $n / d$ & $\mathrm{n} / \mathrm{d}$ & - \\
\hline 08.07 .97 & 1700 & $n / d$ & $\mathrm{n} / \mathrm{d}$ & - \\
\hline 25.07 .97 & 880 & $n / d$ & $\mathrm{n} / \mathrm{d}$ & - \\
\hline 01.08 .97 & 2260 & $n / d$ & $n / d$ & - \\
\hline 05.08 .97 & 1640 & $n / d$ & $\mathrm{n} / \mathrm{d}$ & - \\
\hline 06.08 .97 & 3340 & $\mathrm{n} / \mathrm{d}$ & $n / d$ & - \\
\hline 01.09 .97 & 1620 & $n / d$ & $\mathrm{n} / \mathrm{d}$ & - \\
\hline
\end{tabular}


Tabela 3. Presença (hachurado) e/ou densidade (cél./litro) de microalgas potencialmente tóxicas, nas amostras da área de estudo. Os quadros com hífen (-) indicam ausência do organismo. D. a. = Dinophysis acuminata, D. c. = Dinophysis caudata, D. spp. $=$ Dinophysis spp., P. o. = Prorocentrum obtusum, P. g. = Prorocentrum gracile, P. mic. $=$ Prorocentrum micans, P. $\min =$ Prorocentrum minimum, P. spp. $=$ Prorocentrum spp., G.. spp. $=$ Gymnodinium spp., A. f. $=$ Alexandrium fraterculus, Ps. spp. $=$ Pseudo-nitzschia spp., T. spp. = Thrichodesmium spp.

\begin{tabular}{|c|c|c|c|c|c|c|c|c|c|c|c|c|}
\hline DATA & D. $a$. & D. c. & D. spp. & P.o. & P.g. & P. mic. & P. min. & P. spp. & $\begin{array}{l}G \text {. spp. } \\
\end{array}$ & A. $f$. & Ps. spp. & T. spp. \\
\hline 16.02 .96 & - & - & - & - & - & - & - & 4957 & 4510 & - & - & 902 \\
\hline 16.03 .96 & - & - & - & - & - & - & - & - & - & - & - & - \\
\hline 23.03 .96 & - & - & - & - & - & - & - & - & - & - & - & - \\
\hline 30.03 .96 & - & - & - & - & - & - & - & - & - & - & - & - \\
\hline 13.04 .96 & - & - & - & - & - & - & - & - & - & - & & - \\
\hline 27.04 .96 & - & - & - & - & - & - & - & - & - & - & & - \\
\hline 18.05 .96 & - & - & - & - & - & - & - & - & - & - & 31040 & - \\
\hline 25.05 .96 & - & - & - & - & - & 368 & - & - & - & - & 107456 & - \\
\hline 29.05 .96 & - & - & - & - & - & - & - & - & - & - & 31600 & - \\
\hline 05.06 .96 & - & - & - & - & - & - & - & - & - & - & 3600 & - \\
\hline 15.06 .96 & - & - & - & - & - & - & - & - & - & - & - & - \\
\hline 22.06 .96 & - & - & 40 & - & - & - & - & - & 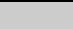 & - & - & - \\
\hline 04.07 .96 & - & - & & - & - & - & - & - & 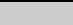 & . & 60 & - \\
\hline 10.07 .96 & - & - & 80 & - & - & 80 & - & - & - & 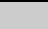 & 360 & - \\
\hline 17.07 .96 & - & 80 & 40 & - & - & - & - & - & - & - & 1160 & - \\
\hline 31.07 .96 & - & - & - & - & - & 1104 & - & - & - & - & 11776 & - \\
\hline 02.08 .96 & - & & - & - & - & & - & - & - & - & 1472 & - \\
\hline 06.08 .96 & - & 600 & 80 & - & - & 600 & - & - & - & - & - & - \\
\hline 13.08 .96 & - & & - & - & - & 368 & - & - & - & . & 73968 & - \\
\hline 23.08 .96 & 451 & & - & - & - & & - & 5410 & 902 & - & - & - \\
\hline 24.08 .96 & - & 200 & 40 & - & - & & - & - & - & - & 3640 & - \\
\hline 07.09 .96 & - & 2206 & & - & - & 1408 & 736 & - & 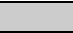 & - & 40480 & - \\
\hline 14.09 .96 & - & & - & - & - & & 1472 & - & - & - & 142784 & - \\
\hline 21.09 .96 & - & 366 & 368 & - & - & - & 1840 & - & 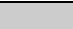 & 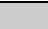 & 19136 & - \\
\hline 28.09 .96 & - & - & & - & - & 7511 & 5633 & - & 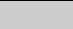 & - & 141964 & - \\
\hline 11.10 .96 & - & 600 & 80 & - & - & 600 & - & - & - & - & - & - \\
\hline 26.10 .96 & - & - & - & - & - & - & - & - & - & - & 19782 & - \\
\hline 29.10 .96 & - & - & - & - & - & - & - & 7210 & - & - & - & - \\
\hline 02.11 .96 & - & - & - & - & - & 1104 & 368 & - & - & - & 33488 & - \\
\hline 10.11 .96 & - & - & - & - & - & 120 & - & - & - & - & 2120 & 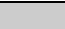 \\
\hline 16.11 .96 & - & - & - & - & - & & - & - & - & - & & - \\
\hline 23.11 .96 & - & - & - & - & - & 2253 & - & - & - & - & 65348 & \\
\hline 30.11 .96 & - & - & - & - & - & - & - & - & - & - & 29072 & 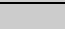 \\
\hline 06.12 .96 & - & - & 40 & - & - & 480 & 240 & - & - & - & 6280 & - \\
\hline 14.12 .96 & - & - & - & & - & - & - & - & - & - & 55200 & - \\
\hline 18.12 .96 & - & - & - & 7662 & 451 & - & - & 9464 & 4510 & - & - & 451 \\
\hline 08.01 .96 & - & - & . & - & - & . & - & - & & - & - & - \\
\hline 15.01 .96 & - & - & - & - & - & . & - & - & & - & & 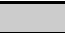 \\
\hline 24.01 .96 & - & - & - & - & - & - & - & - & - & - & & 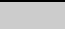 \\
\hline 04.02 .96 & - & - & - & - & - & 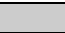 & - & - & - & - & - & - \\
\hline 18.02 .96 & - & - & - & - & - & - & - & - & - & - & 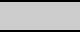 & - \\
\hline 22.02 .96 & - & & 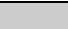 & - & - & 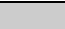 & - & - & 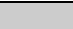 & - & - & - \\
\hline 01.03 .96 & - & & - & - & - & 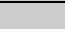 & - & - & - & - & - & - \\
\hline 16.04 .97 & - & - & - & - & - & - & - & 352 & 2464 & 705 & 13380 & 4930 \\
\hline 30.04 .97 & - & - & - & - & - & - & - & 1080 & 800 & 40 & 1480 & - \\
\hline 15.05 .97 & - & - & - & - & - & - & - & 60 & 20 & - & 160 & - \\
\hline 27.05 .97 & - & - & - & - & - & - & - & 100 & 760 & 40 & 100 & - \\
\hline 10.06 .97 & - & - & - & - & - & - & - & - & 60 & - & - & - \\
\hline 24.06 .97 & - & - & - & - & - & - & 40 & 20 & 140 & 20 & 100 & - \\
\hline 08.07 .97 & - & - & - & - & - & - & - & 20 & 240 & - & 740 & - \\
\hline 25.07 .97 & - & - & - & - & - & - & - & - & - & - & - & - \\
\hline 01.08 .97 & - & - & - & - & - & - & 140 & - & 320 & 180 & 20 & - \\
\hline 05.08 .97 & - & - & 60 & - & - & - & - & 60 & 600 & - & 840 & - \\
\hline 06.08 .97 & - & - & - & - & - & - & - & 40 & 380 & - & 300 & - \\
\hline 01.09 .97 & - & - & 40 & - & - & - & - & 80 & 380 & - & 380 & - \\
\hline
\end{tabular}


não publicado). No entanto, pelo menos mais duas espécies deste gênero devem ocorrer na costa catarinense, sendo aconselhável a coleta de amostras vivas, isolamento e cultivo das mesmas para posteriores estudos de fisiologia e toxinologia. O mesmo também é válido para espécies de Prorocentrum.

A carência de dados hidrológicos (circulação, turbulência) e de química da água (nutrientes), paralelos às amostragens limitou a explicabilidade das diferentes composições na assembléia fitoplanctônica, pois não puderam ser feitas análises de correlação entre estes dados. No entanto, utilizando-se os poucos dados ambientais determinados, constatou-se que a razão Diatomáceas : Dinoflagelados apresentou correlação fracamente negativa com a trasparência da água $(r$ $=-0,40 ; n=24 ; P<0,05)$ e fracamente positiva com a temperatura da água $(r=0,32 ; n=24$; $\mathrm{P}<0,10)$. Baixas razões Diatomáceas: Dinoflagelados podem ilustrar uma situação potencialmente perigosa, ou seja, com possibilidade de floração de dinoflagelados, vários dos quais são tóxicos. As correlações acima, embora fracas, sugerem que os dinoflagelados tenderiam a ser mais significativos quando as águas são mais frias e transparentes. A transparência, nesse caso, pode ser indicativo de baixa turbulência (com menor turbulência há menos material em suspensão), situação que favorece a acumulação de dinoflagelados na superfície da água, pois possibilita movimentação flagelar que em situações turbulentas não é significativa.

Comparando-se os resultados deste programa amostral com outros realizados pela FACIMAR na região costeira de Santa Catarina (Macedo, 1997; Lugli 1997) constatou-se que, de maneira geral, a sucessão do fitoplâncton da enseada de Armação do Itapocoroy segue os mesmos padrões dessas outras áreas costeiras. No entanto, particularidades hidrológicas locais, especialmente a menor dinâmica da área, determinam algumas características específicas. A maior estagnação da área pode, como referido acima, favorecer o desenvolvimento de dinoflagelados, entre os quais vários podem ser tóxicos. Analisandose superficilamente os processos regionais, pode-se sugerir que a entrada de frentes frias, aumentando periodicamente a dinâmica hidrológica local, pode prevenir a proliferação e acumulação de dinoflagelados. No entanto, como já verificado em outros ambientes, períodos de estaganção na plataforma continental, seguidos por aumentos bruscos na dinâmica, podem promover proliferação de dinoflagelados em áreas externas e posterior acumulação junto áreas de praia e enseadas, por efeito de processos advectivos (Odebrecht et al., 1995).

Em síntese, os resultados até aqui gerados indicam a necessidade da continuação desta monitorização, com especial atenção nos meses de inverno e primavera (VDM e VAM) e verão (venenos de cianobactérias). $O$ acompanhamento de processos meteorológicos e um estudo criterioso da circulação da água na enseada, são fundamentais para aprimorar esta monitorização. Além disso, é fundamental a expansão desse programa de monitorização para todas as outras áreas de maricultura do estado, o que já está sendo implementado pelo Programa PED (Projeto de Execução Descentralizada) - um convênio entre o Ministério do Meio Ambiente, Governo do Estado de Santa Catarina e FACIMAR/UNIVALI.

\section{LITERATURA CITADA}

Andersen, P. (ed.) 1996. Design and Implementation of some Harmful Algal Monitoring Systems. IOC Technical Series No.44, UNESCO.

Balech, E. 1988. Los Dinoflagelados del Atlântico Sudoccidental. Publicaciones Especiales del Instituto Español de Oceanografia, Ministério de Agricultura, Pesca y Alimentación, Madrid. 310p.

Cardoso, L. de S. 1993. Dinoflagelados da ilha do Arvoredo e da praia de Ponta das Ca- 
nas - SC, Brasil (setembro de 1991 a fevereiro de 1992): considerações taxonômicas e ecológicas. Dissertação de mestrado, Universidade Federal do Rio Grande do Sul. 306p.

Dale, B. \& Yentsch, C.M. 1978. Red tide and paralytic shelfish poisoning. Oceanus, 21, 41-49.

Desikachary, T.V. 1959. Cyanophyta. Indian Council of Agricultural Research, New Delhi. 686p.

Guimarães, S.C.P. \& L.R. Rörig. 1997. Microrganismos associados a florações de Trichodesmium hildebranthii na costa de Santa Catarina - Brasil. Notas Técnicas da FACIMAR (1) 43-47.

Hallegraeff, G.M.; Anderson, D.M.\& A.D. Cembella (eds.) 1995. Manual on Harmful Marine Microalgae. IOC Manuals and Guides $n^{\circ}$ 33. Unesco. 551p.

Lugli, D.O. 1997. Produtividade primária do fitoplâncton na área experimental de maricultura da FACIMAR-UNIVALI (Campus V). Relatório Final de Projeto de Iniciação Científica, ProBIC - UNIVALI. Orientador: Leonardo R. Rörig.

Macedo, C.X. 1997. Produtividade primária das acumulações de diatomáceas de zona de arrebentação da praia de Navegantes, Santa Catarina. Relatório Final de Projeto de Iniciação Científica, ProBIC - UNIVALI. Orientador: Leonardo R. Rörig.

Nielsen, G.E. \& A.M. Bresta. 1984. Guidelines for the measurement of phytoplankton primary production. The Baltic Marine Biologists, pub. $n^{\circ} 1,2^{\text {nd }}$ edition.
Odebrecht, C; Rörig, L.R.; Garcia, V.M.T. \& P.C. Abreu. 1995. Shellfish mortality and a red tide event in Southern Brazil. In: Lassus, P.; Arzul, G.; Erard, E.; Gentien, P. \& C. Marcaillou (eds.). Harmful Algae Blooms. Lavoisier Science Publishers, Paris. pp. 213-218.

Rörig, L.R.; Yunes, J.S.; Kuroshima, K.N.; Schettini, C.A.F.; Pezzuto, P.R. \& L.A.O. Proença. 1998. Studies on the ecology and toxicity of Trichodesmium spp. blooms in Southern Brazilian Coast. In: Reguera, B.; Blanco, J.; Fernández, M.L. \& Wyatt, T [Eds.], Harmful Algae. Xunta de Galicia and Intergorvernmental Oceanographic Comission of UNESCO. pp. 22-25.

Sournia, A. 1978. Phytoplnakton Manual. Monographs on Oceanographic Methodology, UNESCO, Paris. 367p.

Steindinger, K.A. \& J. Williams. 1970. Dinoflagellates. Memories of the Hourglass Cruises, 2: 1-250.

Tomas, C.R. (ed.) 1997. Identifying Marine Phytoplankton. Academic Press, San Diego. 858p.

Utermöhl, H. 1958. Zur vervollkommnung der quantitaiven Phytoplankton Methodik. Mitt. int. Verein. theor. angew Limnol._9:1-38.

Zenebon, O. \& N.P. Pregnolatto. 1992. Memórias técnico-científicas da divisão de bromatologia e química. In: 100 Anos de Saúde Pública, Antunes, J.L.F. (ed.) pp. 173-198. São Paulo: Instituto Adolfo Lutz. 